\title{
Monodirectional Positioning Using Dielectric Elastomers
}

\author{
C. Pagano, M. Malosio, and I. Fassi
}

Institute of Industrial Technology and Automation, CNR Via Bassini 15, Milan, 20133, Italy

\begin{abstract}
The rationales for the use of microsystems are numerous, including the reduction of consumables, a faster response time, the enhanced portability, the higher resolution, and the higher efficiency; moreover their application sectors are numerous. Nevertheless microproducts have still great difficulty in penetrating the market, mainly due to the limits of the fabrication processes. The hybrid approach is suitable for the fabrication of three dimensional microscopic structures but often requires manual contributions, which are time consuming and expensive. In order to overcome these issues, new materials and new techniques for the manipulation of microcomponents, based on innovative principles, have been conceived and have to be further developed. In this paper polymeric smart materials, namely electroactive polymers, have been theoretically and experimentally investigated towards their implementation in the actuation and sensing of positioning and handling devices. The feasibility of a monodirectional positioner has been studied.
\end{abstract}

Keywords: Electroactive polymers, microfactory, microactuator, hybrid microproducts.

\section{Introduction}

Despite the advanced state of conventional actuation technologies, there is an increasing demand for new actuation devices with high power-to-weight ratio, high efficiency and large degree of compliance. In order to fulfill these needs, innovative types of actuators based on smart materials have recently been proposed. They are based on polymeric materials able to change their dimension and/or shape in response to a specific external stimulus such as thermal, chemical, electrical, magnetic or optical. A promising group of such materials is represented by the Electro Active Polymers (EAPs), which exhibit interesting properties, including a controllable high ratio strain versus applied voltage, low specific gravity, high grade of processability, downscalability and, in most cases, low costs [1]. Nevertheless, successful implementation of electroactive polymers mainly depends on the degree of understanding of their behaviour and properties since the selection of the material in the design of smart systems involves considerations of different factors, including the maximum achievable strain, stiffness, spatial resolution, frequency bandwidth and temperature sensitivity. Moreover, it is still essential to develop innovative testing methodologies, consistent material characterization and formulation of advanced theoretical models [2].

In the next section a short introduction on electro-active polymers is presented with particular attention on dielectric elastomers. In the third section the material and the 
realization process of the samples are illustrated. In the fourth section the experimental analyses are described and the results discussed. In the fifth section the concept of an EAP-based planar positioner is presented and, finally, the conclusions are drawn.

\section{Electroactive Polymers}

Electroactive Polymers are polymeric materials whose shape can be modified by applying a voltage to them. The large displacement that can be obtained with EAPs, their low mass, low power and, in some of these materials (ionic polymer) also low voltage, make them very attractive as actuators. Moreover, their mechanical flexibility and ease of processing offer advantages over traditional electroactive materials expanding the options for mechanical configurations. The group of EAPs comprises a wide array of different materials, each characterized by its unique properties and functional abilities [2].

Dielectric elastomers are among the most promising EAPs for many applications including actuators and sensors for the microfactory: they work in a dry environment, can achieve great deformations and support high voltage. When an electric potential difference is applied across the polymeric film, coated with electrodes on both sides, a compressive stress, parallel to the electric field, is generated. This is a well-known phenomenon, known as Maxwell stress, which occurs with all insulators subject to an electric field from deposited electrodes. Nevertheless, only the recent development of soft polymeric thin films with high dielectric breakdown strengths allows the achievement of performances exploitable in actuation devices [3]. EAP operation principle is similar to the electromechanical transduction of a parallel two plate capacitor. When an electric potential difference is applied across the polymeric film, coated with electrodes on both sides, a compressive stress, parallel to the electric field, is generated and, consequently, the material is compressed in thickness and expands in planar directions. In order to permit this deformation the electrodes in an EAP actuator must be compliant because the elastomeric polymers are essentially incompressible in volume and so when the polymeric film is compressed in thickness, it must expand in area [4]. Mechanical actuation can be obtained by the compressive stress which causes a compression along the thickness of the material and the consequent planar expansion of the dielectric elastomer. The electrostatic pressure that compress the film along the thickness direction has been derived via the principle of virtual work assuming that the electrodes are much more compliant than the polymer film itself [5]. This effective pressure generated on the film can be expressed by the following equation:

$$
\mathrm{P}=\varepsilon \varepsilon_{0} \mathrm{E}^{2}=\varepsilon \varepsilon_{0}(\mathrm{~V} / \mathrm{t})^{2}
$$

where $\varepsilon_{0}$ and $\varepsilon$ respectively denote the permittivity of the free space and the dielectric constant of the polymer; $E$ and $V$ denote the applied electric field and voltage, and $t$ is the thickness of the film. Because of the compliance of the electrodes, $\mathrm{P}$ results to be twice higher than the pressure in a rigid parallel plate capacitor [5]. 


\section{Materials and Methods}

EAPs are relatively new materials and, even though their potential applications are spread out in many different sectors, very few EAPs are commercially available and no exhaustive characterization on their electromechanical behaviour exists. For this reason, in order to evaluate their actual limits and potentialities, some experiments have been carried out with a commercial EAP (the 3M acrylic elastomer VHBTM 4910). It is one of the most studied EAP and its electrostrictive behaviour is by now widely known [6]-[10]. The material is a clear and colourless film, with a dielectric constant of 4.7 in the un-stretched state. It is very sticky and can be largely stretched in both directions in the plane of the film. In our experimental setup, firstly, the polymer samples have been pre-stretched up to $700 \%$ along each linear dimension through a circular and adaptable stretching device. Secondly, the film has been packed between a thick and a thin rigid support $(20 \mathrm{~mm} \times 26 \mathrm{~mm})$, both of which present a window of $11 \mathrm{~mm} \times 20 \mathrm{~mm}$ in the centre, in order to obtain a pre-stretched completely constrained sample. In the bare and central region the film is free to deform in consequence of an external electric field. Thirdly, a region with the shape of a stripe $(20 \mathrm{~mm}$ $\mathrm{x} 2 \mathrm{~mm}$ ) on both sides of the film, inside the window and adjacent to one of the bound regions, has been coated with a conductive material. These main components are illustrated in Figure 1. Finally, some markers have been imprinted to one side of the film, through an inked grid-stamp. A sketch and a picture of a sample, together with the reference axes, are shown in Figure 1Figure 2 and Figure 3 respectively. In order to obtain appreciable displacements, the samples have been pre-stretched so that the electric field can be increased without reaching the breakdown voltage [11], in accordance with equation (1).

The shape and the dimension of the electrodes respect to the mechanical constraints have been chosen in order to obtain an almost monodirectional in-plane deformation of the film, along $\mathrm{y}$ axis, whereas the deformation in $\mathrm{x}$ direction can be neglected. This allows the direct transposition of the decrease of the thickness to the deformation along only one direction.

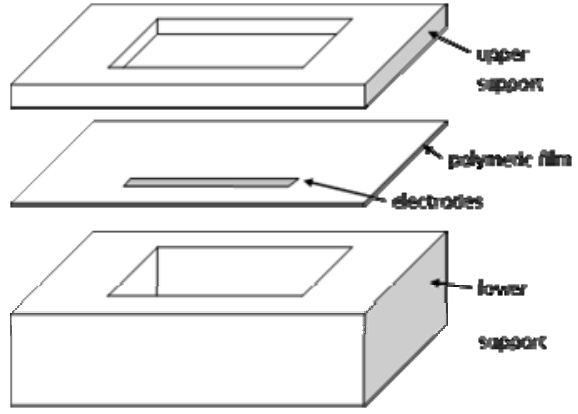

Fig. 1. Exploded view of the experimental sample

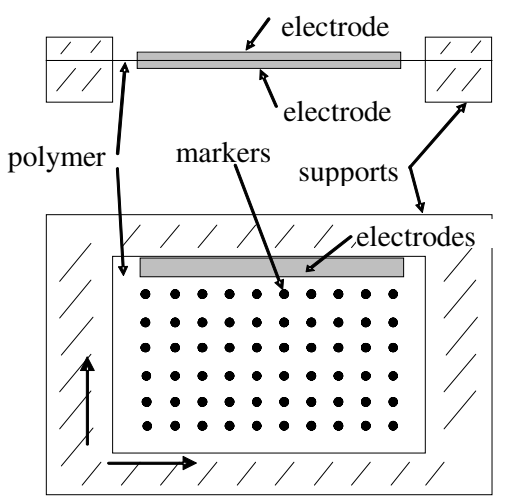

Fig. 2. Sketch of the sample: section view and top view 
The choice of the material for the electrodes is an issue concerning the fabrication of EAP actuators. Indeed, not only have they to be robust enough to endure high electric fields [12], up to hundreds of $\mathrm{kV} / \mathrm{mm}$, but they have also to be very compliant and maintain a good electrical contact when stretched. Graphite powder and silver grease have been tested, in order to obtain highly conductive electrodes. The grease has showed better conductance; hence, Chemtronics Circuit Works ${ }^{\mathrm{TM}}$ silver conductive grease has been preferred and, in some samples, graphite powder has been added to increase the conductivity. Through a suitable vision system the displacement of the markers has been measured in order to quantify the deformation of the polymer when an electric field is applied.

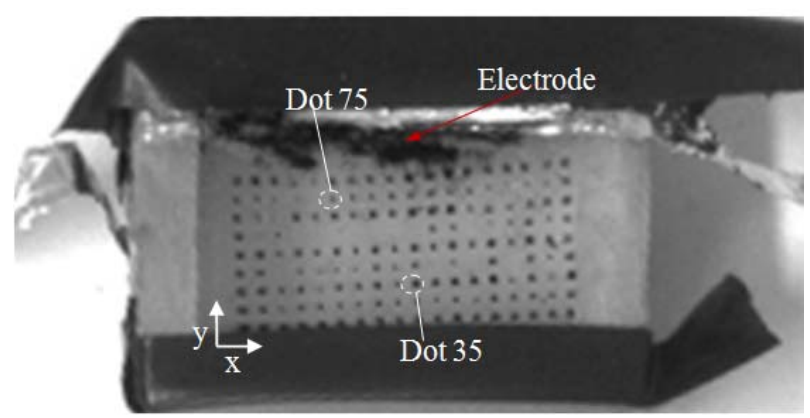

Fig. 3. Picture of a sample with the system reference

Several samples have been prepared and analyzed applying different values of the external voltage, from $1 \mathrm{kV}$ to $6 \mathrm{kV}$ with steps of $1 \mathrm{kV}$. The voltage has been applied to the conductive region of the polymer, coated with the compliant electrode, through a small and thin aluminium wire and the reaction of the central region of the polymer has been recorded through a vision system, equipped with a telecentric objective. The collected images have been elaborated via image processing techniques in order to obtain information about the position and the displacement of the markers and, therefore, the planar deformation of the polymer. The charge/discharge cycles procedure is here described: firstly the lowest voltage $(1 \mathrm{kV})$ has been applied and turned off for few seconds and then, turned on again for 5-7 times. The same procedure has been followed applying 2, 3, 4, 5 and finally $6 \mathrm{kV}$ and between two following charge/discharge cycles a time interval of approximately 2 minutes have been waited to identify possible residual deformations, permit to overcome transitories and avoid failures due to significant loads for long periods of time [13]. All the process has been recorded by the vision system with a 30 frames-per-second rate. Moreover all the charge/discharge cycles have been analysed at once, tracing the displacements of the dots with respect to their positions in the very first image (before applying $1 \mathrm{kV}$ ) and over the entire period of time. The displacement along $\mathrm{x}$ and $\mathrm{y}$ of a dot near the electrodes (dot 75 in Figure 3) and one far away from the electrodes (dot 35 in Figure 3) has been plotted versus the time in Figure 4 and Figure 5 respectively. 


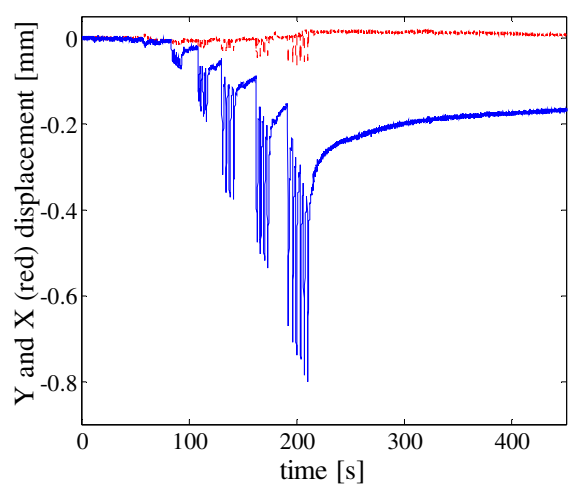

Fig. 4. X-Y displacement of dot 75 in Figure 3 Fig. 5. X-Y displacement of dot 35 in Figure 3 vs. time

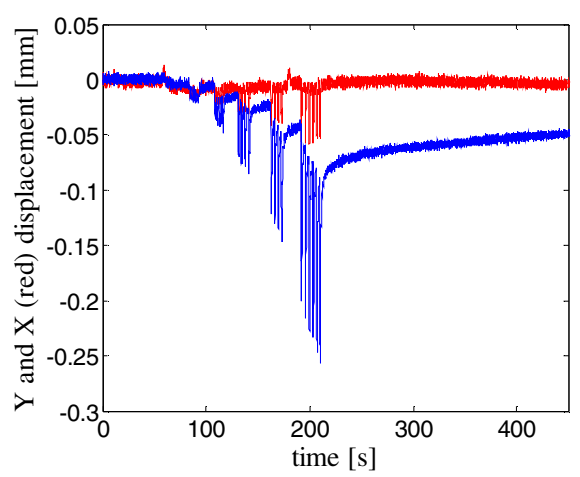

vs. time

In both the figures the deformation cycle due to the $1 \mathrm{kV}$ tension is not appreciable (because the deformations are comparable to the camera resolution), while deformations due to higher tensions can be clearly identified. In both the figures the displacements along the $y$ direction (perpendicular to the main axis of the electrode) are much bigger than the ones in the $x$ direction; this confirms the behaviour expected from the layout of the electrodes.

Furthermore, comparing the magnitude of the displacements in Figure 4 and Figure 5 it is evident that, as expected, the closer to the electrodes the dot, the bigger the displacement. Finally, a residual deformation is evident in both the plots, and, as expected, a greater residual deformation is associated with the dot nearer to the electrodes (dot 75 in Figure 4).

In Figure 6 small movements are notable along $x$ almost only in the upper part of the plot where the movement along the y direction are significantly small and effects of mechanical constraints can much more influence the deformation of the polymer. Further analyses of the displacements along $x$ will be carried out in order to estimate the influence of boundary effects.

In Figure 7 the maximum measured displacement in the $y$ direction of two samples with different thickness $(28 \mu \mathrm{m}$ and $62 \mu \mathrm{m})$ has been plotted versus the applied voltage.

The thickness of the samples has not been experimentally measured, but evaluated from the magnitude of the mechanical pre-stretch. Indeed, since the Poisson ratio of the material is equal to 0.49 [14], it has been considered incompressible and the thickness of the sample has been calculated according to the following formula:

$$
\mathrm{x}^{2}+\mathrm{y}^{2}+\mathrm{z}^{2}=\text { constant }
$$

where $\mathrm{x}, \mathrm{y}$ and $\mathrm{z}$ are the three dimensions of the film.

The displacement increases in a non linear way when the applied voltage is increased and the results obtained for both the samples have been interpolated with a second order polynomial curves, shown in Figure 7. The consistency of the interpolating curves with the results seems to be in agreement with equation (1) even if 


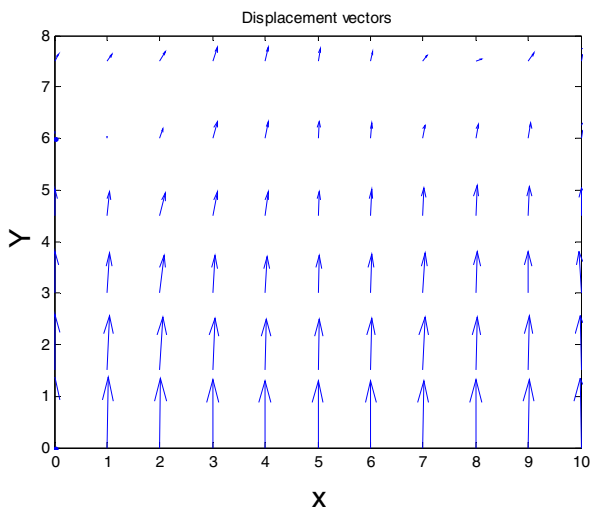

Fig. 6. The arrows refer to the displacement field of the sample, their direction and module represent the maximum displacement of the reference points on the sample

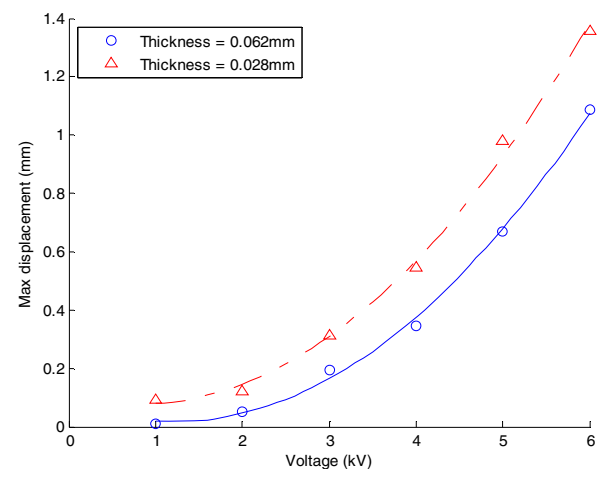

Fig. 7. Absolute values of the maximum displacement along the $y$ direction versus the applied voltage

further investigations on the deformation along the $z$ direction have to be carried out to fully understand the behaviour of the materials. Moreover, this behaviour is in agreement with the hypothesis according to which the electrical compression in the $z$ direction (perpendicular to the plane of the polymeric film) is mainly transformed in a stretch along the $y$, whereas the $x$ displacement is strongly limited by the bounds and the asymmetric shape of the electrode region. Finally, it can be noticed that the two curves are deformed along the y coordinate due to the different thickness of the film of the samples. Indeed, the voltage applied to both the samples is the same (from $1 \mathrm{kV}$ to $6 \mathrm{kV}$ ), but, due to the thickness, the electric field strength is different, according with equation (1). Finally, Figure 7 shows that displacements bigger than $1 \mathrm{~mm}$ can be obtained with $6 \mathrm{kV}$ and that a range of about one order of magnitude can be achieved varying the voltage between 1 and $6 \mathrm{kV}$.

\section{Planar Positioner}

Electroactive polymers are very promising in a variety of sectors and, in particular for applications in the Microfactory. Indeed, due to the light load and the reduced space available, the devices required at small scale have specific needs, such as lightness, compactness, precision and rapidity, which are essential requirements for microactuators together with common needs, such as suitable force and stroke.

The idea behind the actuated samples can be directly converted into a monodimensional linear actuator. In order to realise a position-controlled device two opposite couples of electrodes are required, so that the actuation of each of them can recover residual deformations and histeretical behaviour of the material, due to a previous activation of the opposite one.

Moreover, a more advanced bi-dimensional micropositioning actuator, made of dielectric elastomer has been conceived. In Figure 8 a sketch of it is drawn: a rigid 
square-shaped frame holds a single piece of dielectric polymer (white in the figure), which is partially coated on both sides with 4 compliant electrodes (active regions) represented with the 4 grey squares.

The active regions expand when an external voltage is applied, they compress in thickness and, as a consequence, compress the central passive region of polymer, which is no subject to any electric field. Hence, if a component is placed in the central region of the polymer it can be moved, operating on the electrodes.

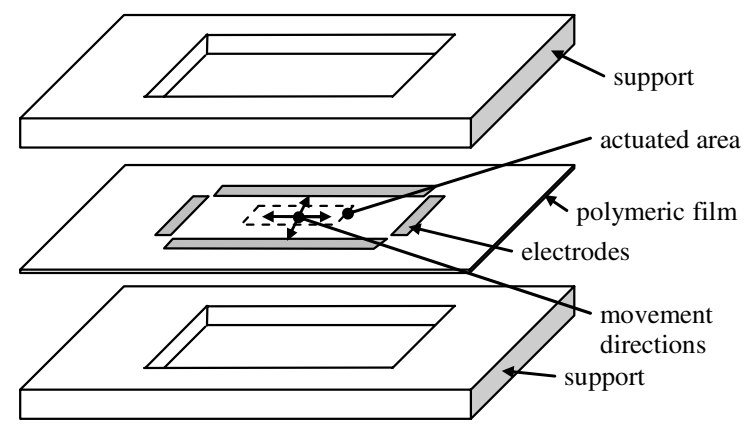

Fig. 8. Sketch of a potential configuration of a micropositioning made of dielectric elastomer

This configuration allows the activation of one or more active regions at time and also the recovery of residual deformations and the precise control of the position of the central region in the plane in two dimensions for planar positioning applications.

\section{Conclusions}

Although a lot of potential applications of electroactive polymers could enable many innovations in several fields, their diffusion is still limited by the lack of clear understanding of all their properties. Indeed their structure-property response interrelations is complex and a further issue is due to the sensitivity of electroactive polymers to their fabrication conditions, time and temperature dependent effects, and nonlinearity of their coupled mechanical, physical and chemical properties.

In this paper the electro-mechanical behaviour of an acrylic EAP has been studied and a few physical properties, such as the strain for different input loads and the presence of a residual deformation have been observed. Moreover an application of the polymer in the microfabrication of hybrid MEMS has been introduced, taking into account the results obtained. Further analyses will be carried out in order to characterize and model the hyperelastic behaviour of the material both under a theoretical and an experimental point of view. On the basis of the experimental results, a modelbased control system will be studied, in order to realise a positioning device with submicrometric precision as required by the application sector. 


\section{References}

[1] De Rossi, D., et al.: NTS Inc., pp. 119-135 (2004)

[2] Vinogradov, A., et al.: Mater. Res. Soc. Symp. Proc., 889 (2006)

[3] Bar-Cohen, Y.: SPIE Press, Bellingham (2004)

[4] Pelrine, R.E., et al.: Adv. Mater. 12/16 (2000)

[5] Pelrine, R.E.: Sensor Actuator A 64, 77-85 (1998)

[6] Jung, K., et al.: Smart Mater. Struct. 16, S288-S294 (2007)

[7] Kofod, G., et al.: Proc. of SPIE, vol. 4329, pp. 141-147 (2001)

[8] Pelrine, R.E., et al.: Science 287, 836 (2000)

[9] Wissler, M., Mazza, E.: Sensors and Actuators A 134, 494-504 (2007)

[10] Carpi, F., et al.: Sensors and Actuators A 107, 85-95 (2003)

[11] Kofod, G., et al.: J. of Intelligent Material Systems and Structures 14(12), 787-793 (2003)

[12] Santer, M.J., Pellegrino, S.: Proc. of the 45th AIAA/ASME/ASCE/AHS/ASC Structures, Palm Springs, California, April 19-22 (2004)

[13] Plante, J.S., Dubowsky, S.: Smart Mater. Struct. 16, S227-S236 (2007)

[14] 3M VHBTM Tapes Technical 